Tohoku J. exp. Med., 1983, 141, Suppl., 343-353

\title{
Characteristics Associated with Diabetic Retinopathy in Nauruans
}

\author{
H. King, P. Zimmet, R. Taylor, B. Balkau, L.R. Raper, \\ J. Borger and W. Herriot \\ WHO Collaborating Centre for the Epidemiology of \\ Diabetes Mellitus, Royal Southern Memorial Hospital, \\ Melbourne, Australia
}

\begin{abstract}
King, H., Zimmet, P., Taylor, R., Balkau, B., Raper, L.R., Borgee, J. and Herriot, W. Characteristics Associated with Diabetic Retinopathy in Nauruars. Tohoku J. exp. Med., 1983, 141, Suppl., 343-353 — A recent epidemiological survey of the whole adult Micronesian poplulation of Nauru has confirmed that Nauruans, along with Pima Indians, suffer the highest rate of abnormal glucose tolerance yet recorded. To establish the morbid effects of hyperglycaemia in this population, all responders to the diabetes survey were concurrently examined for diabetic retinopathy. In diabetic subjects, the crude prevalence of retinopathy was $24 \%$. Specific rates were determined at various levels of the following characteristics : age, 2 hour post-load plasma glucose, body mass index, duraton of diabetes and systolic blood pressure. Prevalence was found to rise with increasing 2 hour plasma glucose and duration, to fall with increasing body mass index and to have a quadratic relationship with age and systolic blood pressure. The multiple logistic regression model was used to determine whether the selected characteristics were significant in increasing the risk of retinopathy. Body mass index and systolic blood pressure did not contribute significantly to this risk after controlling for age. Increasing 2 hour plasma glucose significantly increased the risk of retinopathy, and duration of disease was the strongest predictor variable. This study shows that the consequences of hyperglycaemia in this Micronesian population are comparable to those already documented in European and American Indian communities.— micronesia; diabetic retinopathy; duration; hyperglycaemia
\end{abstract}

Few population-based epidemiological studies have assessed the prevalence of retinopathy in Caucasoid or non-Caucasoid diabetics. Certain non-Caucasoid communities have been found to suffer very high prevalence rates of diabetes mellitus (DM) according to internationally defined criteria ${ }^{1,2,3)}$. However, apart from the Pima Indians ${ }^{45}$ ) it has not been conclusively established whether these populations are suffering from DM itself, or have hyperglycaemia after an oral glucose tolerance load, without suffering from the specific microvascular complica-

Address for reprints : Professor Paul Zimmet, Head and Principal Investigator, World Health Organization Collaborating Centre for the Epidemiology of Diabetes Mellitus, Royal Southern Memorial Hospital, PO Box 185, Caulfield South, 3162, Victoria, Australia. 
tions of DM seen in Caucasoids.

In order to resolve the question as to the consequences of hyperglycaemia in these populations, diabetic retinopathy is a useful marker of the diabetic disease state. Dorf et al.5) have found in a population-based study of Pima Indians over the age of 15 years, that the prevalence of retinopathy in diabetics was $18 \%$, and that duration of disease was the most strongly associated factor. Prevalence of retinopathy rose from $3 \%$ among newly diagnosed diabetics to $48 \%$ among those with a duration of 10 years or more.

The Micronesian population of Nauru share with the Pimas the dubious distinction of suffering the higiest prevalence of DM in the world ${ }^{6)}$. They are also a well-defined island community ideally suited for the study and elucidation of this issue with respect to Pacific populations.

In January, 1982, a total adult population survey of Nauru was undertaken, and all subjects were concurrently examined for diabetic eye disease. The prevalence of DM and of diabetic retinopathy was determined, and the association of retinopathy with age, 2 hour post-load plasma glucose ( $2 \mathrm{~h} P G)$, body mass index (BMI), duration of diabetes and systolic blood pressure (SBP) was examined.

\section{Materials and Methods}

All Nauruans present on the island at the time of the survey formed the target population. An estimate of 1984 persons aged 20 years and over was obtained from the 1980 elctoral roll. The presence of a person's name on the electoral roll was a necessary precursor to voting. Voting is taken seriously by Nauruans, and the roll was therefore considered to be an accurate estimate of the population. Of these 1984 persons, 69 were either overseas, or too sick to attend, leaving an eligible population of 1915 . Of these, 1583 attended the survey giving a response rate of $83 \%$.

All eligible subjects were given an invitation on the day prior to the survey and advised to fast from midnight. At the survey site, fasting, and $2 \mathrm{~h} \mathrm{PG}$ samples were taken, the glucose load of $75 \mathrm{~g}$ being given orally. Anthropometric measurements were made, and blood pressure $(\mathrm{mmHg})$ was measured in the sitting position using a random zero sphygmomanometer. A questionnaire concerning diabetic and hypertensive status and treatment, smoking, alcohol consumption, and family history of disease was answered. Both direct and indirect ophthalmoscopy was performed by our ophthalmologists (JB and WH) after the dilation of both pupils. The ophthalmologist was not aware of the glucose tolerance status of the subject being examined.

DM was diagnosed in accordance with current $W H O$ criteria $^{73}$, i.e. as plasma glucose 200 $\mathrm{mg} / 100 \mathrm{ml}(11.1 \mathrm{mmol} / \mathrm{liter})$, or more, two hours after a $75 \mathrm{~g}$ oral glucose load. In the absence of a two-hour sample, a fating plasma glucose level of $140 \mathrm{mg} / 100 \mathrm{ml}(7.8 \mathrm{mmol} /$ liter) or more was taken as diabetic, and all known diabetics were included. The duration of disease for the known diabetics was taken to be the time since the first diagnosis.

Retinopathy was also defined according to accepted international criteria ${ }^{\mathrm{B}}$. For the purpose of this analysis, retinopathy was considered as a binary variable (presence or absence), presence being one or more microaneurysm, haemorrhage or exudate in either eye, or the presence of neovascularisation. 


\section{Results}

The prevalence of diabetes in the Nauruan population over the age of 20 years was $24 \%$, rising to $46 \%$ in those aged 35 years and over (Zimmet et al. in preparation). Fig. 1 shows the prevalence of retinopathy in diabetics according to duration of disease. Prevalence rose steadily in both sexes, from less than $10 \%$ in newly diagnosed cases, to more than $50 \%$ in those with a duration of diabetes of 10 years or more.

The trend was similar in the two sexes, and the sexes were combined for further univariatd analysis. Figs. 2-6 show prevalence of retinopathy at various levels of the five selected independent variables. Prevalence rose with both $2 \mathrm{~h}$ PG (Fig. 2) and duration (Fig. 3). The relationship with age appeared quadratic (Fig, 4), perhaps because those with the more severe retinopathy-producing disease would be less likely to live to an advanced age. Prevalence ef retinopathy fell with increasing BMI (Fig. 5), and it might be postulated that the more severe the disease, the leaner the subject, either due to disease or treatment. Level of systolic blood pressure did not seem to have a strong effect on prevalence of retinopathy (Fig. 6). Such a trend as there was seemed similar to that observed for age.

In order to examine the independence of the selected characteristics relative to each other, a correlation matrix was constructed (Table 1). It is desirable from a statistical point of view to have low correlations amongst the independent or predictor variables. Table 1 shows this to be the case for these data, the highest correlation was 0.32 between BMI and SBP. BMI was also correlated weakly and

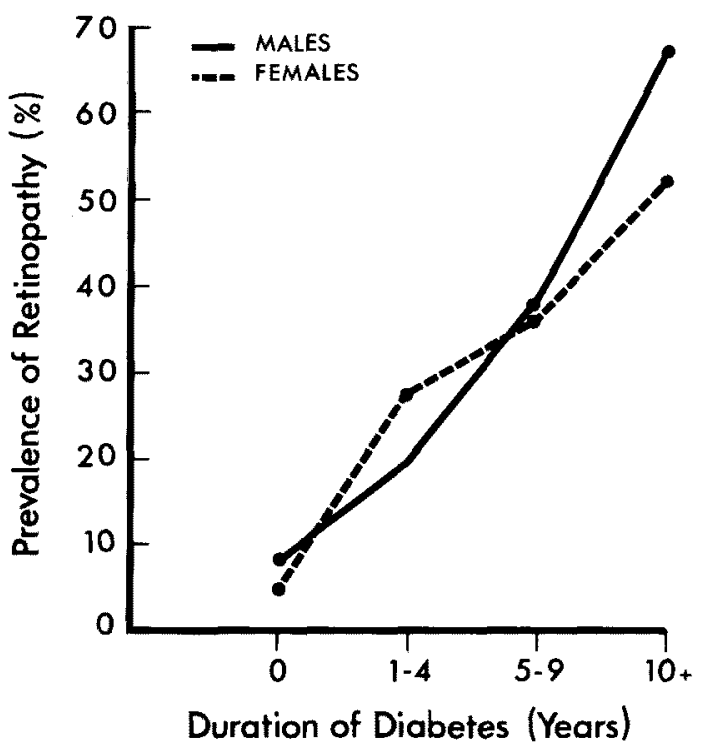

Fig. 1. The relationship between duration of diabetes and retinopathy. 


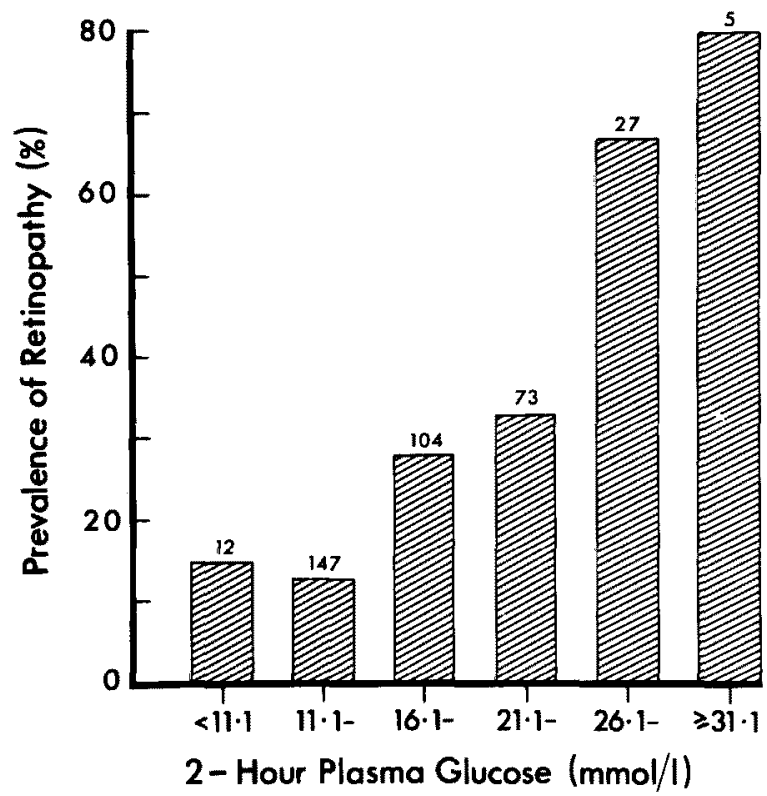

Fig. 2. The relationship between plasma glucose and retinopathy (sexes combined).

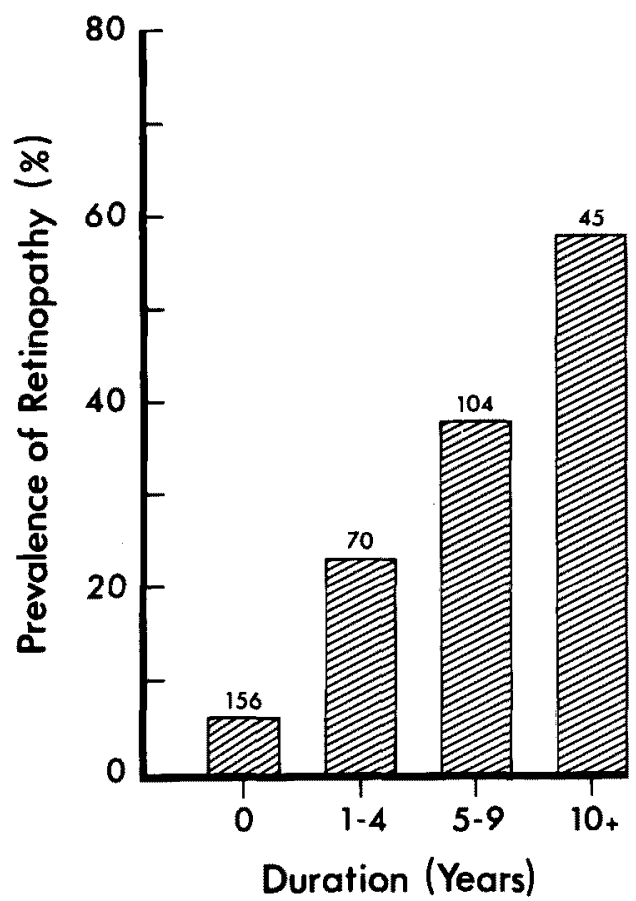

Fig. 3. The melationship between duration of diabetes and retinopathy (sexes combined). 


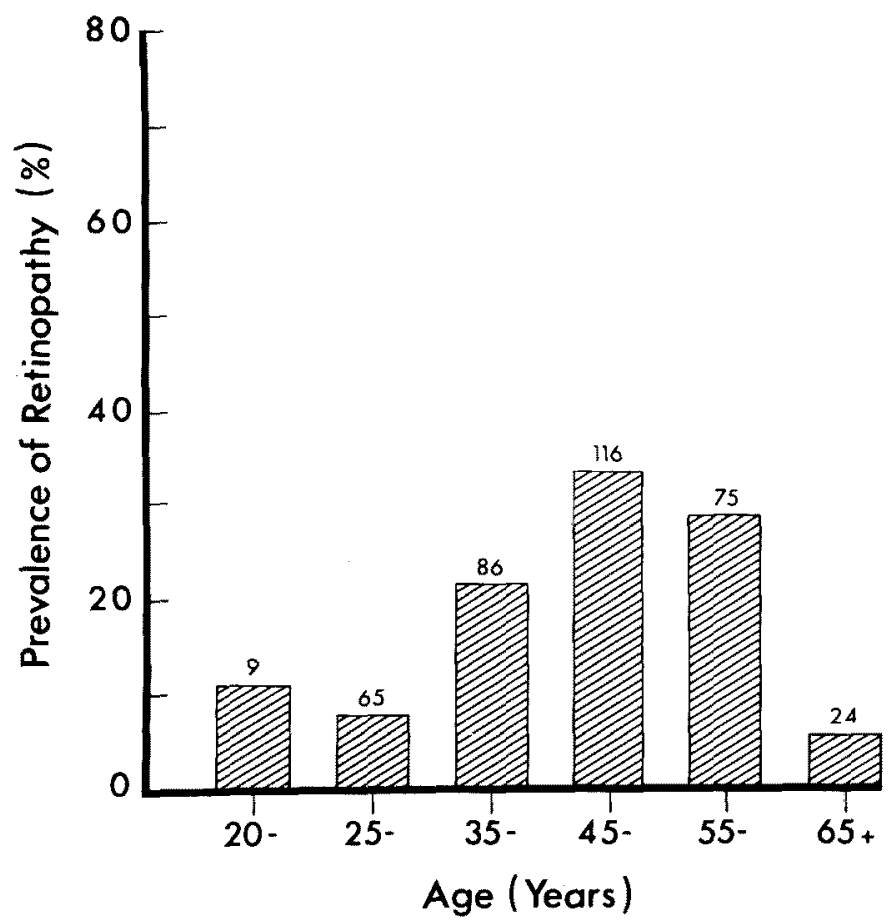

Fig. 4. The relationship between age and retinopathy (sexes combined).

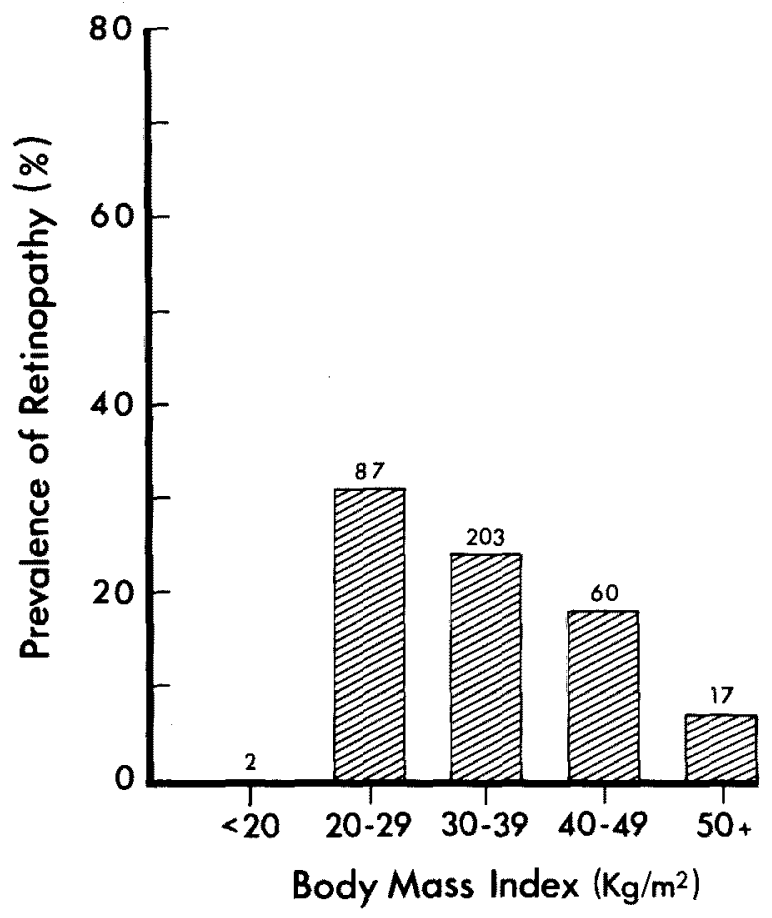

Fig. 5. The relationship between body mass index and retinopathy (sexes combined). 


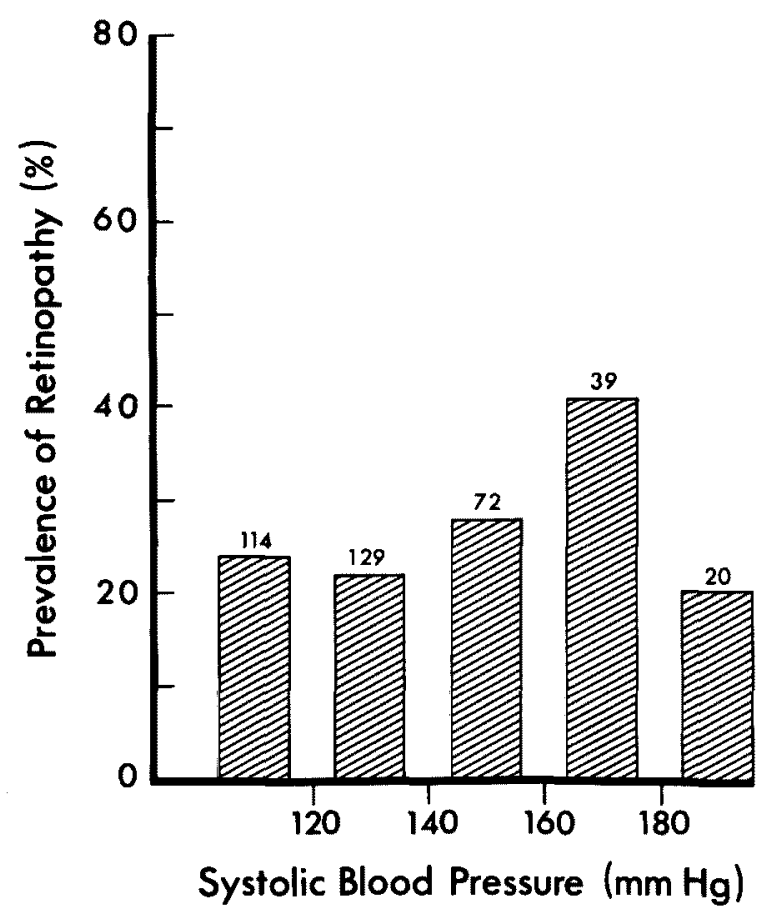

Fig. 6. relationship between systolic blood pressure and retinopathy (sexes combined).

TABLE. 1. Correlation coefficients between selected characteristics, sexes combined

\begin{tabular}{lrrcc}
\hline & Age & 2h PG & BMI & Duration \\
\hline 2h PG (mmol/liter) & 0.10 & - & - & - \\
BMI (kg/m $\left.{ }^{2}\right)$ & -0.26 & -0.20 & - & - \\
Duration (years) & 0.13 & 0.17 & -0.12 & - \\
SBP (mmHg) & 0.09 & -0.11 & 0.32 & -0.08 \\
\hline
\end{tabular}

negatively with age and $2 \mathrm{~h} \mathrm{PG.} \mathrm{There} \mathrm{was} \mathrm{little} \mathrm{correlation} \mathrm{between} \mathrm{duration}$ and any of the other variables, or between any other combination.

Mean values of the selected characteristics were then determined in those with and without retinopathy in each sex seperately (Table 2). There was little difference in age, BMI and SBP between groups with or without retinopathy in either sex. However, there was a highly significant difference in mean $2 \mathrm{~h} \mathrm{PG}$ in both sexes ( $p<0.01$ for males, $p<0.001$ for females) and the difference in mean duration between the two groups was the most significant of all factors examined in both sexes $(p<0.001)$. Univariate analysis suggests, therefore, that of the five variables selected, duration of of disease is the one most strongly associated with retinopathy, with $2 \mathrm{~h} P \mathrm{PG}$ also contributing a significant effect.

To determine the combined effect of the variables, the multiple logistic 
TABLE. 2. Mean士 S.E.M. of selected characteristics according to sex and retinopathy status

\begin{tabular}{lrlrr}
\hline \multirow{2}{*}{ Retinopathy } & \multicolumn{2}{c}{ Males } & \multicolumn{2}{c}{ Females } \\
\cline { 2 - 5 } & \multicolumn{1}{c}{ Present } & Absent & Present & Absent \\
\hline Age & $48.5 \pm 1.5$ & $47.4 \pm 1.2$ & $48.8 \pm 1.4$ & $44.6 \pm 1.0^{*}$ \\
2h PG (mmol/liter) & $19.9 \pm 0.7$ & $17.0 \pm 0.5 \dagger$ & $21.2 \pm 1.0$ & $17.4 \pm 0.4 \ddagger$ \\
BMI (kg/m $)$ & $32.8 \pm 0.7$ & $33.7 \pm 0.6$ & $33.9 \pm 1.0$ & $36.7 \pm 0.6^{*}$ \\
Duration of diabetes & $6.9 \pm 0.7$ & $2.7 \pm 0.3 \ddagger$ & $8.5 \pm 1.0$ & $2.6 \pm 0.3 \ddagger$ \\
(years) & $136.3 \pm 6.6$ & $133.3 \pm 2.4$ & $136.2 \pm 4.3$ & $134.1 \pm 2.0$ \\
SBP (mmHg) & $* p<0.05 ; \dagger p<0.01 ; \ddagger p<0.001 ;$ Student's $t$ test.
\end{tabular}

regression model (see Appendix) was fitted to males and females separately, whose $2 \mathrm{~h}$ PG level was equal to or exceeded $7.8 \mathrm{mmol} / \mathrm{liter}$. The analysis was carried out using the GLIM program9). Individuals were classified into six age-groups (20-24, 25-34, 35-44, 45-54, 55-64, 65- years and over), and 2h PG, BMI, duration and SBP were treated as continuous variables. Allowance was made for the possibility of a non-linear trend in the prealence of retionpathy with age by combining individuals into age groups, and always including a different constant term in the model for each age-group.

The model with the four variables was compared with models which also included squared terms and interactions between the variables. These comparisons were made using the difference of the deviance between the models, and comparing them with the chi-square distribution") (see Appendix). As these equations were not significantly better in predicting the presence or absence of retinopathy, the simpler model was selected for further analysis.

Extreme data points in this chosen model which, if included, would markedly effect the variable co-efficients and fit of the model were found using the diagnostic technique of Pregibon ${ }^{10)}$. These were excluded from the final analysis because they had the effect of swamping the general trend of the rest of the data. In all, seven males and nine females were excluded for this reason, leaving 155 males and 188 females.

The coefficients in the logistic regression models are given in Table 3. As measures of the fit of the models, the deviance, which has a chi-square distribution with the given number of degrees of freedom, indicates that the model for both males and females are acceptable ones, while the 'entropy' of the models, as defined by Efron ${ }^{11)}$, is .45 and .46 respectively. These values can be interpreted as the " $R$ " values for the models (see Appendix for details).

To determine which variables, and combination of variables were the best predictors of retinopathy, all $2^{4}$ possible models with combinations of the four variables were analysed. All models allowed for the six age group constants. 
TABLE. 3. Coefficients and other characteristics of the multiple logistic model to predict retinopathy status

\begin{tabular}{|c|c|c|c|c|}
\hline \multicolumn{5}{|c|}{ Co-efficients (Standard error) } \\
\hline & \multicolumn{2}{|c|}{ Males $(n=155)$} & \multicolumn{2}{|c|}{ Females $(n=188$ ) } \\
\hline Duration (years) & \multicolumn{2}{|c|}{$.45(.094)$} & \multicolumn{2}{|c|}{$33(.075)$} \\
\hline $2 \mathrm{~h} \mathrm{PG}$ (mmol/liter) & \multicolumn{2}{|c|}{$.21(.069)$} & \multicolumn{2}{|c|}{$.081(.043)$} \\
\hline $\mathrm{SBP}(\mathrm{mmHg})$ & \multicolumn{2}{|c|}{$.015(.011)$} & \multicolumn{2}{|c|}{$.012(.011)$} \\
\hline BMI $\left(\mathrm{kg} / \mathrm{m}^{2}\right)$ & \multicolumn{2}{|c|}{$.022(.051)$} & \multicolumn{2}{|c|}{$.056(.040)$} \\
\hline Age Group 1 & -16 & $(51)$ & -12 & $(27)$ \\
\hline Age Group 2 & -9.8 & (51) & -12 & $(28)$ \\
\hline Age Group 3 & -11 & (51) & -4.2 & $(27)$ \\
\hline Age Group 4 & -10 & (51) & -3.4 & $(27)$ \\
\hline Age Group 5 & 9.9 & (51) & -5.4 & (27) \\
\hline Age Group 6 & -19 & $(52)$ & -12 & $(37)$ \\
\hline \multicolumn{5}{|c|}{ Measures of the fit of the models* } \\
\hline Deviance & \multicolumn{2}{|c|}{93.9} & \multicolumn{2}{|c|}{101.4} \\
\hline Degree of freedom & \multicolumn{2}{|c|}{145} & \multicolumn{2}{|c|}{178} \\
\hline Entropy & \multicolumn{2}{|c|}{.45} & \multicolumn{2}{|c|}{.46} \\
\hline
\end{tabular}

* See Appendix for definitions.

TABLE. 4. Comparison of models with one, two, three, and four variables included. The age group variables have been included

\begin{tabular}{lccc}
\hline & Deviance & $\begin{array}{c}\text { Degrees } \\
\text { of } \\
\text { freedom }\end{array}$ & $\boldsymbol{\chi}^{2 *}$ \\
\hline Males $(n=155)$ & 107.4 & 148 & $13.65 \dagger$ \\
$\quad$ Duration & 96.6 & 147 & 2.7 \\
Duration, 2h PG & 94.1 & 146 & .2 \\
Duration, 2h PG, SBP & 93.9 & 145 & \\
Complete model, 4 variables & & & \\
Females $(n=188)$ & 108.3 & 181 & $6.9^{*}$ \\
Duration & 104.1 & 180 & 2.7 \\
Duration, 2h PG & 102.7 & 179 & 1.3 \\
Duration, 2h PG, BMI & 101.4 & 178 & \\
Complete model, 4 variables & & & \\
\hline
\end{tabular}

* The $\chi^{2}$ value is the observed value for a likelihood ratio test. Each model is compared with the complete model when all four variables are included. See Appendix for methods.

Table 4 shows the deviances for the best model for each sex with one, two, three and four variables included, the best model being the one with smallest deviance. It should be noted that we have not used the "standardized coefficients" to 
determine which variable is the most important, as this can be misleading due to correlations between variables ${ }^{12}$.

Comparing models for both males and females, by taking the differences of the deviances, there is no significant difference between the model with only duration and $2 \mathrm{~h} \mathrm{PG}$, and the model including all four variables. Thus, these two variables together predict the retinopathy status as well as all four variables. Duration is the most important predictor, SPB and BMI differ in their order of importance in males and females, but neither variable has much to add to either model.

\section{DiscUSSION}

The data reported here have been analysed both by univariate methods, and also by the application of the multiple logistic regression model. Both methods of analysis show that in both sexes, duration of diabetes is the factor most strongly associated with the presence of retinopathy, with $2 \mathrm{~h} \mathrm{PG}$ also being significantly associated. After controlling for age, BMI and SBP had no useful predictive value isolated from the two most important factors.

These findings are substantially in accord with data collected on the Pimas) and Oklahoma Indians ${ }^{14)}$ and strengthen the likelihood of the truth of the apparent associations. Furthermore, the consistency of these Pacific data with similar material collected from other populations supports the notion that Pacific islanders do indeed suffer from diabetes in a form comparable to that found in Caucasoid and other more closely studied communities rather than merely demonstrating hyperglycaemia as an innocent biochemical trait.

The cross-sectional nature of this material must not be overlooked, and it will only be with prospective studies, now underway in the Pacific, that aetiological conclusions may be drawn with confidence. However, the consistent findings in this and other studies ${ }^{5,13,14)}$ that not only duration of diagnosed disease, but also 2h PG level at the time of examination are significantly associated with the presence of retinopathy highlight the desirability of the control of hyperglycaemia in diabetics. This applies not only in Westernized societies with already wellestablished control programmes, but also in regions such as the Pacific.

\section{APPENDIX}

\section{The multiple logistic regression model}

The multiple logistic regression mocdl predicts

$$
p=\text { probability of retinopathy }
$$

given, in this case, the age group, duration, $2 \mathrm{~h} \mathrm{PG}, \mathrm{SBP}$ and BMI of an individual. This model is in the from 


$$
\begin{aligned}
\log _{e}[ & p /(1-p)] \\
= & \alpha_{i}+\beta_{1} \times(\text { duration }) \\
& +\beta_{2} \times(2 \mathrm{~h} \mathrm{PG}) \\
& +\beta_{3} \times(\mathrm{SBP})+\beta_{4} \times(\mathrm{BMI})+\text { Error }
\end{aligned}
$$

Where $\alpha_{1 i}, \beta_{1}, \beta_{2}, \beta_{3}, \beta_{4}$ are constants determined by use of the computer package, GLIM $^{10)}$. There are six constants $\alpha_{i}$, corresponding to the age group of an individual.

The deviance of the model, as output by GLIM ${ }^{10)}$ is found from the ratio of the $\log$ likelihoods of a current model, $L_{c}$ and the full model, $L_{f}$, when all observations are perfectly fitted. The deviance is defined by

Deviance $=-2 \log _{e}\left(L_{c} / L_{f}\right)=-2 \log _{e}\left(L_{c}\right)+2 \log _{e}\left(L_{f}\right)$

A small deviance indicates a good fit, and the value can be compared with a chisquare distribution with degrees of freedom equal to the difference in the number parameters estimated by the two models. The full model has $n$ parameters estimated, one parameter for each observation. To compare models 1 and 2 , we use the difference in the deviances,

Deviance (1)-Deviance $(2)=2 \log _{e}\left(L_{1}\right)-2 \operatorname{kog}_{e}\left(L_{2}\right)$

and compare this with the chi-square distribution with degrees of freedom equal to the difference in the number of parameters estimated.

Another measure of the goodness of fit is defined by Efron ${ }^{11}$, as the entropy of a model. It uses the log likelihood of the current model, $L_{e}$ and compares it with the $\log$ likelihood of the model, $L$, when no variables are fitted when $p$ is predicted by the average prevalence.

$$
\text { Entropy }=\left[-2 \log _{e}(L)+2 \log _{e}\left(L_{c}\right)\right] /\left[-2 \log _{e}(L)\right]
$$

and thus the entropy is the proportional reduction in residual variation in going from a crude model to a model using predictor variables. It can be interpreted in the same way as the " $R^{2}$ " value of multiple regression.

\section{Acknowledgments}

These studies were undertaken as joint collaborative projects between the Health Department of the Governments of Western Samoa and Nauru, the World Health Organization, and the Royal Southern Memorial Hospital. The study and data analysis were carried out with the generous financial support of World Health Organization and NIH grant 1RO1 AM 25446-02.

We thank Miss R Swan for help in the preparation of the manuscript, and Mrs A Malpass for preparing the figures.

\section{References}

1) Zimmet, P. (1979) Epidemiology of diabetes and its macrovascular manifestations in Pacific populations: the medical effects of social progress. Diabetes Care, 2, 144.

2) Zimmet, P., Faaiuso, S., Ainui, J., Whitehouse, S., Milne, B. \& DeBoer, W. (1981) The prevalence of diabetes in the rural and urban Polynesian population of Western Samoa. Diabetes, 30, 45.

3) West, K.M. (1978) Epidemiology of Diabetes and Its Vascular Complications. Elsevier, New York. 
4) Bennett, P.H., LeCompte, P.M., Miller, M. \& Rushforth, N.B. (1976) Epidemiological studies of diabetes in the Pima Indians. Recent Prog. Horm. Res., 32, 333-376.

5) Dorf, A., Ballintine, E.J., Bennett, P.H. \& Miller, M. (1976) Retinopathy in Pima Indians. Diabetes, 25, 554.

6) Zimmet, P., Taft, P., Guinea, A., Guthrie, W. \& Thomas, K. (1977) The high prevalence of diabetes mellitus on a Central Pacific Island. Diabetologia, 13, 111.

7) WHO Expert Committee of diabetes Mellitus (1980) Technical Report Series 646, WHO Geneva.

8) Bennett, P.H. (1979) Recommendations on the standardization of methods and reporting of tests for diabetes and its microvascular complications in epidemiologic studies. Diabetes Care, 2, 98.

9) Baker, R.J. \& Nelder, J.A. (1978) The GLIM System Release 3, Generalised Linear Interactive Modelling, oxford: Numerical Algorithim Group.

10) Pregibon, D. (1981) Logistic regression diagnostics. Amer. Stat., 9, 705.

11) Efron, B. (1978) Regression and ANOVA with zero-one data: measures of residual variation. J. Amer. Stat. Ass., 73, 361, 113.

12) Mosteller, F. \& Tukey, J.W. (1977) Data Analysis and Regression. Addison-Wesley.

13) Zimmet, P., Taylor, R. \& Bennett, P. (1972) Diabetic microangiopathy in Pacific populations. Presented at the International Symposium on Diabetic Microangiopathy, Osaka, Japan, March, 1982.

14) West, K.M., Erdreich, L.J. \& Stober, J. (1980) Detailed study of risk factors for retinopathy and nephropathy in diabetes. Diabetes, 20, 501-508. 